\title{
Sensory Experience Alters Cortical Connectivity and Synaptic Function Site Specifically
}

\author{
Claire E. J. Cheetham, Martin S. L. Hammond, Clarissa E. J. Edwards, and Gerald T. Finnerty \\ Medical Research Council Centre for Neurodegeneration Research, King's College London, London SE5 8AF, United Kingdom
}

\begin{abstract}
Neocortical circuitry can alter throughout life with experience. However, the contributions of changes in synaptic strength and modifications in neuronal wiring to experience-dependent plasticity in mature animals remain unclear. We trimmed whiskers of rats and made electrophysiological recordings after whisker cortical maps have developed. Measurements of miniature EPSPs suggested that synaptic inputs to layer $2 / 3$ pyramidal neurons were altered at the junction of deprived and spared cortex in primary somatosensory cortex. Whole-cell recordings were made from pairs of synaptically connected pyramidal neurons to investigate possible changes in local excitatory connections between layer $2 / 3$ pyramidal neurons. The neurons were filled with fluorescent dyes during recording and reconstructed in three dimensions using confocal microscopy and image deconvolution to identify putative synapses. We show that sensory deprivation induces a striking reduction in connectivity between layer $2 / 3$ pyramidal neurons in deprived cortex without large-scale, compensatory increases in the strength of remaining local excitatory connections. A markedly different situation occurs in spared cortex. Connection strength is potentiated, but local excitatory connectivity and synapse number per connection are unchanged. Our data suggest that alterations in local excitatory circuitry enhance the expansion of spared representations into deprived cortex. Moreover, our findings offer one explanation for how the responses of spared and deprived cortex to sensory deprivation can be dissociated in developed animals.
\end{abstract}

Key words: synaptic; plasticity; experience-dependent plasticity; somatosensory; cortex; whisker

\section{Introduction}

The neocortex is not hardwired but reorganizes with persistent changes in the sensory periphery during development (Hensch, 2004; Feldman and Brecht, 2005) and adulthood (Fox and Wong, 2005). Cortical map reorganization induced by brief periods of sensory deprivation is a popular experimental paradigm with which to study experience-dependent plasticity. Plasticity is commonly greatest in layer $2 / 3(\mathrm{~L} 2 / 3)$ at the junction of cortex that has lost its sensory input (deprived) and cortex that has retained its sensory input (spared) (Fox, 1992; Gilbert and Wiesel, 1992; Diamond et al., 1994; Glazewski and Fox, 1996). Distinct mechanisms with different temporal profiles drive changes in sensory responses to deprived and spared inputs (Fox, 2002). In somatosensory cortex, responses to deprived inputs depress rapidly (over a few days) after whisker deprivation during adolescence, but this effect becomes less prominent when deprivation is started in adult animals (Glazewski and Fox, 1996; Wallace and Fox, 1999). In contrast, responses to spared sensory inputs are enhanced. This enhancement develops more slowly than the depression of responses in deprived cortex of adolescent animals but can be elicited in adulthood (Glazewski et al., 1996).

\footnotetext{
Received July 24, 2006; revised Jan. 31, 2007; accepted Feb. 20, 2007.

The work was funded by the Wellcome Trust, King's College Hospital Medical Research Trust (C.E.J.C.), and the Medical Research Council (C.E.J.E.). G.T.F. is a Wellcome Trust Senior Fellow in the Clinical Sciences. We thank Barry Connors, John Parnavelas, and Ben Philpot for helpful comments.

Correspondence should be addressed to Gerald T. Finnerty at the above address. E-mail: g.finnerty@iop.kcl.ac.uk. D0I:10.1523/JNEUROSC1.5143-06.2007

Copyright $\odot 2007$ Society for Neuroscience $\quad$ 0270-6474/07/273456-10\$15.00/0
}

The relative contributions of changes in synaptic strength and modifications in neuronal wiring to either enhancement or depression of sensory responses in neocortex have not been fully elucidated (Chklovskii et al., 2004). Structural changes in axons (Darian-Smith and Gilbert, 1994; Kossut and Juliano, 1999) and dendritic spine turnover (Trachtenberg et al., 2002; Zuo et al., 2005b) have been reported after sensory deprivation, but the new synaptic partners acquired after axonal growth or new spine formation are not always identified.

In vitro studies of the effects of experience-dependent plasticity on synaptic strength in neocortex have commonly focused on vertical intracortical pathways or long-distance (hundreds of micrometers to millimeters) horizontal connections that cross cortical columns (Rioult-Pedotti et al., 1998; Finnerty et al., 1999; Allen et al., 2003; Bender et al., 2006; Clem and Barth, 2006). Changes in the strength of short-range (less than a few hundred micrometers) horizontal connections within cortical columns have attracted less attention. Pyramidal neurons in L2/3 and L5 do not connect randomly with neighboring pyramidal neurons but form local excitatory networks (Song et al., 2005; Yoshimura et al., 2005). These networks may be an important substrate for sensory processing that occurs on a subcolumnar basis (Brumberg et al., 1999) [e.g., angular tuning responses of whiskers (Andermann and Moore, 2006)]. The output from local networks in L2/3 provides much of the excitatory drive for the lateral, intracortical spread of activity in vivo (Petersen et al., 2003). Hence, local excitatory connections are in a position to play a key role in regulating the size of cortical maps. Here we report that alter- 
ations in sensory input, which start after cortical maps have developed, induce a marked reduction in local connectivity between L2/3 pyramidal neurons in deprived cortex of developed animals without large-scale modifications in connection strength. In contrast, connectivity in local excitatory networks within spared cortex is unchanged, but connection strength is potentiated.

\section{Materials and Methods}

Whisker trimming. All procedures were performed in accordance with the United Kingdom Animals (Scientific Procedures) Act 1986. We trimmed bilaterally the dorsal three $(\mathrm{A}-\mathrm{C})$ or the ventral two (D-E) rows of rats' whiskers flush with the skin every day. Whisker trimming or shamtrimming started at postnatal day 19 (P19), which is after the L2/3 whisker map has developed (Stern et al., 2001), and continued for 11-41 d.

Brain slice preparation and recording. On the day of electrophysiological recording, all whiskers were trimmed acutely by an assistant to blind experimenters to the rat's sensory history. The rat's brain was cooled rapidly by transcardial perfusion with ice-cold dissection buffer comprising the following (in mM): 108 choline-Cl, $3 \mathrm{KCl}, 26 \mathrm{NaHCO}_{3}, 1.25$ $\mathrm{NaHPO}_{4}, 25$ D-glucose, $3 \mathrm{Na}$ pyruvate, $2 \mathrm{CaCl}_{2}$, and $1 \mathrm{MgCl}_{2}$ (bubbled with $\left.95 \% \mathrm{O}_{2} / 5 \% \mathrm{CO}_{2}\right)$. Brain slices $300-400 \mu \mathrm{m}$ thick were cut at $65^{\circ}$ to the midline (Fig. $1 \mathrm{~A}$ ), incubated in artificial CSF containing $120 \mathrm{~mm}$ $\mathrm{NaCl}, 3 \mathrm{~mm} \mathrm{KCl}, 23 \mathrm{~mm} \mathrm{NaHCO}, 1.25 \mathrm{~mm} \mathrm{NaHPO}_{4}, 10 \mathrm{~mm}$ D-glucose, $2 \mathrm{mM} \mathrm{CaCl}_{2}$, and $1 \mathrm{mM} \mathrm{MgCl}_{2}$ bubbled with $95 \% \mathrm{O}_{2} / 5 \% \mathrm{CO}_{2}$ at $35^{\circ} \mathrm{C}$ for $45 \mathrm{~min}$ and then maintained at room temperature until transferred to the recording bath, which was heated to $36-37^{\circ} \mathrm{C}$. Bright-field illumination was used to identify brain slices containing whisker barrels (Fig. $1 \mathrm{~A}$ ). Neurons in layer $2 / 3$ of the $\mathrm{C}$ or $\mathrm{D}$ barrel columns were visualized using infrared differential interference contrast. Recording pipettes (4-7 M $\Omega$ ) contained the following: $130 \mathrm{~mm} \mathrm{KMeSO}$, $8 \mathrm{~mm} \mathrm{NaCl}, 2 \mathrm{~mm} \mathrm{KH} \mathrm{PO}_{4}, 2$ mm D-glucose, 10 mm HEPES, $1 \mathrm{~mm}$ Alexa Fluor 488 (AF488) or $1 \mathrm{~mm}$ Alexa Fluor 568 (AF568) (Invitrogen, Carlsbad, CA), and $3 \mathrm{mg} / \mathrm{ml}$ biocytin.

Paired recordings. The loose-seal current injection protocol was designed to minimize the risk of missing small-amplitude or facilitating connections. It comprised four trials of five pulses (5 ms, $1-10 \mathrm{nA}, 20$ $\mathrm{Hz}$ ). Trials were repeated every $5 \mathrm{~s}$. If there was a definite or possible response in the "postsynaptic" neuron, then the "presynaptic" neuron that had the loose seal made on it was repatched with a second pipette containing the internal solution described above and whole-cell recording was established. A pair of neurons was termed "connected" when both cells were recorded in whole-cell mode and action potentials in one neuron evoked EPSPs in the other neuron (see Figs. $1 B, 5 D$ ). Connections were denoted "reciprocal" when the connection was bidirectional. Statistical analysis of connectivity only uses connections confirmed with whole-cell recordings. It does not incorporate the numbers of reciprocal connections. Connectivity data from initial nonblind experiments were pooled with data from recordings performed blind to the sensory history of the rats because there was no significant difference between the results for the two groups (data not shown).

The presynaptic neuron was stimulated to fire a train of eight action potentials by repetitive injection of brief ( $2-3 \mathrm{~ms}$ ), depolarizing current pulses and the postsynaptic potential was recorded (Multiclamp 700A; Molecular Devices, Sunnyvale, CA). The amplitude of the depolarizing current pulses was adjusted to ensure that only one action potential was fired by the presynaptic neuron per current pulse. Action potentials were filtered at $10 \mathrm{kHz}$ and postsynaptic responses at $3 \mathrm{kHz}$. All signals were digitized at $20 \mathrm{kHz}$. We fitted a single-exponential curve to a $3-10 \mathrm{~ms}$ period before EPSP onset and a second function, $a+b \times t \times e^{-c \times t}+$ $d \times t \times e^{-g \times t}$, to the EPSP over a 3-20 ms window encompassing the EPSP peak (Fig. 1B). EPSP amplitude was estimated from the function generated by subtracting the extrapolated baseline curve from the EPSP curve. Steady-state amplitude was defined as the mean of the last four responses in the train. Data acquisition and analysis were performed with custom-written programs in Labview (National Instruments, Austin, TX) and Matlab (MathWorks, Natick, MA). Neuronal location was confirmed after recording by overlaying $5 \times$ images of the slice and the flu-
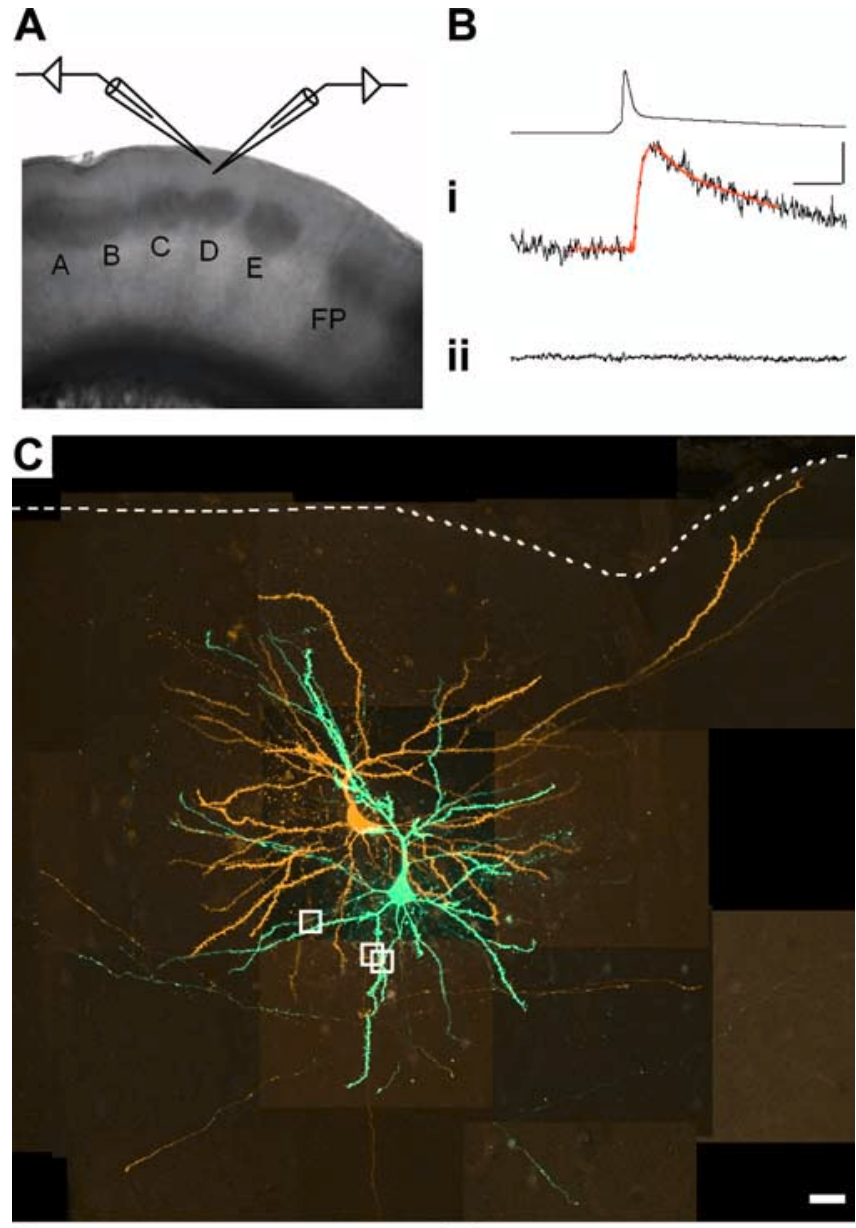

D

\section{E}
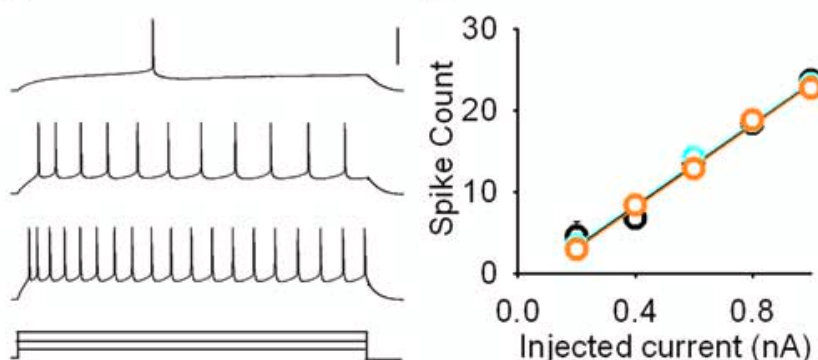

Figure 1. Excitatory transmission between synaptically connected layer $2 / 3$ pyramidal neurons. $\boldsymbol{A}$, Low-power $(2 \times)$ image of a brain slice. Whisker barrel columns are labeled A-E. FP denotes forepaw barrel columns. $\boldsymbol{B}$, Action potential: $\boldsymbol{i}$, evoked EPSP (single trial) in a synaptically connected pair of pyramidal neurons with baseline and EPSP fits overlaid in red; $i i$, averaged response (30 trials) with ionotropic glutamate receptors blocked. Calibration: 80 and 0.4 $\mathrm{mV}, 5 \mathrm{~ms}$. C, Montage of maximum intensity projections from confocal z-stacks of synaptically connected neurons filled with AF568 (orange; presynaptic) or AF488 (green; postsynaptic). White dashed line denotes pia. White boxes indicate sites of putative synapses. Scale bar, 20 $\mu \mathrm{m}$. $\boldsymbol{D}$, Regular spiking pattern recorded in response to $500 \mathrm{~ms}$ pulses of depolarizing current $[+230$ (rheobase), +400 , and $+600 \mathrm{pA}]$. Calibration: $60 \mathrm{mV}$. $\boldsymbol{E}$, Mean number of action potentials elicited by increasing current injection in control (black; $n=33$ ), deprived (cyan; $n=30$ ), and spared (orange; $n=35$ ) cortex.

orescent dye-filled neurons. Septal width was estimated by measuring the distance between the D and E barrels (maximum of $120 \mu \mathrm{m}$ ), which is the largest interbarrel gap with this slice orientation (Fig. $1 A$ ). We excluded paired recordings if either neuron in the pair was $<60 \mu \mathrm{m}$ from the center of the supraseptal region between $\mathrm{C}$ and $\mathrm{D}$ barrel columns because L2/3 pyramidal neurons above L4 septa have different circuitry (Kim and Ebner, 1999; Brecht et al., 2003) and experience-dependent plasticity has 
different effects on circuitry in barrel columns and the septal projection to L2/3 during development (Shepherd et al., 2003).

Miniature EPSPs. Whole-cell recordings of miniature EPSPs (mEPSPs) were made at resting membrane potential with $1 \mu \mathrm{M}$ tetrodotoxin and $100 \mu \mathrm{m}$ picrotoxin (Tocris Bioscience, Bristol, UK) in the recording bath. One neuron per slice was studied for the longer-duration deprivation protocol (22-34 d). Neurons in the C, D, and E barrel columns of each slice were recorded for the shorter-duration deprivation protocol (13-20 d). The data acquisition parameters were the same as used for unitary EPSPs. We used shape criteria derived from measurements of evoked unitary EPSPs (see above, Paired recordings) (Fig. $1 B$ ) to identify mEPSPs in our traces. The criteria required a depolarizing potential with amplitude $>62.5 \mu \mathrm{V}$ (2.5 times the root mean square of baseline noise) (Clements and Bekkers, 1997), a time-to-peak $<5 \mathrm{~ms}$, and a rate of decay after peak that was between $2 \%$ of peak mEPSP amplitude per ms and (50/time to peak)\% of peak mEPSP amplitude per millisecond. EPSPs that overlapped (collided) were considered to be separate when the second EPSP started $\geq 1 \mathrm{~ms}$ after the peak of the first EPSP. EPSP amplitudes were measured using custom-written software (Labview) under manual control. A minimum of 50 mEPSPs were measured per cell.

Confocal microscopy. Slices were fixed in $4 \%$ paraformaldehyde for 30 min, mounted with Gel/Mount (Biomeda, Foster City, CA) and imaged on a Zeiss (Oberkochen, Germany) LSM510 META confocal microscope with a $63 \times, 1.2$ numerical aperture C-Apochromat water immersion objective. Pinhole diameters (maximum, 1 Airy unit) were adjusted to give identical optical slice thicknesses for AF488 and AF568 tracks. Medium resolution $z$-stacks ( $\leq 120 \mu \mathrm{m}$ ) were acquired initially. Regions in which synapses were suspected were then reimaged at high resolution (voxel, $0.07 \times 0.07 \times 0.2 \mu \mathrm{m}$ ). Datasets were deconvolved with AutoDeblur (AutoQuant, Troy, NY) and imaged with Imaris (Bitplane, $\mathrm{Zu}-$ rich, Switzerland) to visualize synapses. The optical performance of the confocal microscope (scan head alignment, correction for chromatic aberrations, $z$-axis drift) combined with deconvolution was tested with 0.1 and $4 \mu \mathrm{m}$ Tetraspeck fluorescent microspheres (Invitrogen) mounted similarly to the brain slices. The peak fluorescence signal from AF488 and AF568 fluorophores was within $0.1 \mu \mathrm{m}$ in the image plane and within the same optical section. Criteria for synapses were based on previously reported electron microscopic reconstructions of excitatory synapses onto L2/3 pyramidal neurons (Peters and Kaiserman-Abramof, 1969) convolved with the point spread function of emitted light. Putative synapses were defined to occur when there was $>0.40 \mu \mathrm{m}$ overlap of a presynaptic varicosity with a postsynaptic spine or dendritic shaft in the $x-y$ plane and the overlap extended over a minimum of four contiguous $0.2 \mu \mathrm{m}$ slices in the confocal $z$-stack (supplemental video, available at www. jneurosci.org as supplemental material).

Statistics. All results are given as mean \pm SEM unless noted. Mean mEPSP amplitudes underwent a reciprocal transformation, and mean mEPSP frequencies underwent a logarithmic transformation to normalize the data and stabilize their variances before performing a two-way ANOVA. We modeled the dependence of connectivity, mean mEPSP amplitude, and unitary EPSP amplitude on age and deprivation status under the generalized linear model framework (McCullagh and Nelder, 1989) using the "stats" Package implemented in R (R Project for Statistical Computing, http://www.r-project.org/) with models of the following general form: $g[E(Y)]=\alpha+\beta \times$ age $+\gamma \times \mathrm{DEP}+\delta \times \mathrm{SP}+\varepsilon \times$ age $\times \mathrm{DEP}+\zeta \times$ age $\times \mathrm{SP}$, where $E(Y)$ is the expected value of connectivity, mean mEPSP amplitude, or unitary EPSP amplitude, $g$ is a link function, DEP is a dummy variable $(1=$ deprived, otherwise 0$)$ for deprived $\mathrm{L} 2 / 3$ neurons, and $\mathrm{SP}$ is a dummy variable $(1=$ spared, otherwise 0 ) for spared L2/3 pyramidal neurons. For simplicity, interactions between deprivation status and the duration of deprivation were modeled using age, which equals the duration of deprivation plus $19 \mathrm{~d}$. The link function and distribution family, e.g., binomial, Gaussian, are specified for each model in Results.

The distributions of control and spared unitary EPSP amplitudes were well fitted by gamma distributions whose probability density functions had the following general form: $g(x ; r, \lambda, \mu)=\lambda^{r} \times(x-\mu)^{(r-1)} \times$ $\exp (-\lambda \times(x-\mu)) / \Gamma(r)$, where $x$ is the unitary EPSP amplitude, $r$ and $\lambda$ are constants $>0, \mu$ is the offset constant, and $\Gamma$ is the gamma function.
The best fits were found by minimizing the Anderson-Darling statistic $\left(A^{2}\right)$, which places greater emphasis on the tails of distributions than the Kolmogorov-Smirnov test (Stephens, 1986), and was implemented in Matlab (MathWorks). The null hypothesis for the two-sample Anderson-Darling test was that control and spared unitary EPSP amplitudes were two random samples drawn from the same parent population, which we assumed also had a gamma distribution. The hypothesis was tested by finding the gamma distribution that maximized the probability that the spared and control unitary EPSP amplitudes were both drawn from it. The probability for each gamma distribution tested required the distribution of the Anderson-Darling statistic, which we found with Monte Carlo simulations that generated distributions with 100,000 data points. To verify our Monte Carlo distribution results, we compared them with published tables (Stephens, 1986) and found that they agreed to three significant figures.

\section{Results}

\section{Reduced connectivity in deprived cortex}

We altered the sensory input to somatosensory cortex of rats after the whisker cortical map in L2/3 has developed (Stern et al., 2001) by trimming bilaterally the dorsal three $(\mathrm{A}-\mathrm{C})$ or the ventral two (D-E) rows of whiskers for 11-37 d (mean, $23 \mathrm{~d}$ ). We then prepared brain slices that permitted visualization of the whisker barrel rows in somatosensory cortex (Fig. $1 A$ ). Sham-trimmed rats served as controls. We looked for evidence of altered connectivity by searching for pairs of synaptically connected L $2 / 3$ pyramidal neurons (Fig. $1 B, C$ ) that were within the same barrel column and close to the boundary between deprived and spared cortex using the loose-seal technique (Feldmeyer et al., 1999) (see Materials and Methods) (see Figs. 5A, B, 6A).

We performed 2683 loose-seal tests. The frequency of connections in control cortex was 1:35 (32 connections, 1117 looseseals). A total of $22 \%$ ( 7 of 32 ) of these connections were reciprocal. Connectivity in spared cortex (frequency of 1:45, 20 connections identified from 902 loose seals; 3 (15\%) reciprocal) was not changed compared with controls $\left(\chi^{2}=0.60 ; \mathrm{df}=1 ; p=\right.$ $0.44)$. In contrast, connectivity in deprived cortex was dramatically reduced (frequency of $1: 83 ; 8$ connections identified from 664 loose seals; $0 \%$ reciprocal $)$ compared with controls $\left(\chi^{2}=\right.$ $4.50 ; \mathrm{df}=1 ; p=0.034)$. Addition of the glutamatergic antagonists CNQX $(20 \mu \mathrm{M})$ and AP-5 $(50 \mu \mathrm{M})$ to the recording bath blocked evoked EPSPs ( $n=8$ connections) (Fig. $1 B$ ). There was no difference between the groups in the age of the animals studied, duration of sensory deprivation, or passive membrane properties (Table 1).

The effect of age and the duration of deprivation (age minus 19 d) on connectivity in spared and deprived cortex over our recording period (P30-P60) was explored under the generalized linear model framework. We first used the following model: logit $[E$ (connectivity $)]=\alpha+\beta \times$ age $+\gamma \times \mathrm{DEP}+\delta \times \mathrm{SP}$, with a logit link function and the binomial family (see Materials and Methods). The results suggested that connectivity between $\mathrm{L} 2 / 3$ pyramidal neurons was reduced in deprived cortex $(\gamma=$ $-0.951 \pm 0.399 ; p=0.017 ; z=-2.38)$ but not in spared cortex $(\delta=-0.272 \pm 0.286 ; p=0.342 ; z=-0.95)$ and that connectivity was not modified by age in isolation $(\beta=-0.003 \pm 0.019$; $p=0.887 ; z=-0.14)$. We could not determine whether the effect of deprivation increased with the duration of deprivation by incorporating interaction terms into this model because of collinearity. Therefore, we analyzed the connectivity data using a second "interaction" model: logit $[E$ (connectivity) $]=\alpha+\gamma \times$ $\mathrm{DEP} \times$ age $+\delta \times \mathrm{SP}$. The interaction model did not result in a significant improvement in fit to the data (null deviance, 123; non-interaction model, residual deviance, 116; interaction 
Table 1. Effects of sensory deprivation on layer $2 / 3$ pyramidal neurons

\begin{tabular}{llcc}
\hline & Control & Deprived & Spared \\
\hline Age (postnatal days) & $42(36-48)$ & $41(37-45)$ & $45(35-46)$ \\
Trim duration (days) & $23(17-29)$ & $22(18-26)$ & $26(16-27)$ \\
Presynaptic resting membrane potential $(\mathrm{mV})$ & $-76(-78$ to -72$)$ & $-75(-79$ to -74$)$ & $-73(-78$ to -70$)$ \\
Postsynaptic resting membrane potential $(\mathrm{mV})$ & $-76 \pm 1$ & $-77 \pm 1$ & $-77 \pm 1$ \\
Postsynaptic input resistance $(\mathrm{M} \Omega)$ & $59 \pm 3$ & $14.1 \pm 0.7$ & $57 \pm 4$ \\
Postsynaptic membrane time constant $(\mathrm{ms})$ & $300(250-360)$ & $12.3 \pm 1.4$ & $14.5 \pm 0.6$ \\
Rheobase $(\mathrm{pA})$ & $6.8 \pm 0.3$ & $250(210-300)$ & $280(210-400)$ \\
Input- output slope in Figure $1 E$ (action potentials per $\mathrm{nA}) \times 10^{-2}$ & $6.5 \pm 0.3$ & $6.1 \pm 0.3$ \\
\hline
\end{tabular}

Age, trim duration, and passive membrane properties are from recordings of pairs of synaptically connected pyramidal neurons. Normally distributed data are reported as mean \pm SEM. Non-normal data are given as median (interquartile range). One-way ANOVA showed no difference in postsynaptic resting membrane potential ( $\left.p=0.43 ; F_{(2,60)}=0.86 ; n=63\right)$, postsynaptic input resistance $\left(p=0.58 ; F_{(2.58)}=0.54 ; n=61\right)$, postsynaptic membrane time constant $(p=$ $\left.0.31 ; F_{(2,57)}=1.19 ; n=60\right)$, or input- output slope $\left(p=0.22 ; F_{(2,95)}=1.53 ; n=98\right)$. One-way ANOVA on ranks revealed no difference in age $(p=0.95 ; H=0.10 ; \mathrm{df}=2 ; n=61)$, trim duration $(p=0.95 ; H=0.10 ; \mathrm{df}=2 ; n=61)$, presynaptic resting membrane potential $(p=0.36 ; H=2.04 ; \mathrm{df}=2 ; n=63)$, or rheobase $(p=0.54 ; H=1.24 ; \mathrm{df}=2 ; n=93)$.

model, residual deviance, 116) but was consistent with a progressive decrease in connectivity in deprived cortex with age $(\gamma=$ $-0.024 \pm 0.010, p=0.017, z=-2.38 ; \alpha=-3.461 \pm 0.179, p<$ $0.001, z=-19.3)$. The expected frequency of connections in deprived cortex at P30 was 1:66. This decreased to 1:132 at P60. Our findings indicate that sensory deprivation results in a selective loss of local excitatory connectivity in deprived cortex close to spared cortex. The statistical model using DEP as an explanatory variable effectively assumed that the effect of deprivation was independent of age and was as good at explaining the data as the model incorporating DEP $\times$ age. Hence, a reasonable interpretation of the statistical models is that a large part of the reduction in connectivity between L2/3 pyramidal neurons in deprived cortex had developed by P30 and that an additional reduction in connectivity probably occurred between P30 and P60.

\section{Miniature EPSPs are larger in spared and deprived cortex}

The reduced connectivity that we found indicated that pyramidal neurons in deprived cortex received less synaptic input from neighboring pyramidal neurons. Moreover, we found no compensatory modifications in either the excitability or the passive membrane properties of $\mathrm{L} 2 / 3$ pyramidal neurons (Fig. $1 D, E, \mathrm{Ta}-$ ble 1). Hence, we surmised that this combination of changes would manifest as a reduction in measures of synaptic input onto deprived pyramidal neurons.

Rats' whiskers were trimmed for 21-34 d, and brain slices were prepared. We blocked action potentials and fast inhibition to isolate mEPSPs (Fig. 2A) (see Materials and Methods), which are thought to comprise postsynaptic responses to release of single vesicles of excitatory neurotransmitter (Fatt and Katz, 1952). mEPSPs were recorded with the same parameters used for paired recordings to facilitate comparison with evoked unitary EPSPs of small amplitude. The mean frequency of mEPSPs in either spared cortex $(10 \pm 1 \mathrm{~Hz} ; p=0.72, t$ test; $n=10$ neurons $)$ or deprived cortex ( $9 \pm 1 \mathrm{~Hz} ; p=0.27, t$ test; $n=10$ neurons $)$ was not different from control cortex ( $10 \pm 1 \mathrm{~Hz} ; n=10$ neurons) (Fig. $2 B)$. The frequency of mEPSPs that we found is similar to values reported previously for recordings at physiological temperatures in younger animals (Hardingham and Larkman, 1998; Simkus and Stricker, 2002). However, the mean amplitude of mEPSPs in both spared cortex $(0.18 \pm 0.02 \mathrm{mV} ; p=0.016, t$ test; $n=10$ neurons $)$ and deprived cortex $(0.19 \pm 0.02 \mathrm{mV} ; p=0.030, t$ test; $n=10$ neurons) was greater than in control cortex $(0.13 \pm 0.01$ $\mathrm{mV} ; n=10$ neurons) (Fig. $2 C-F)$. The empirical distribution function of the mean amplitude of mEPSPs in deprived cortex was displaced to the right relative to controls ( $p=0.031$, Kolmogorov-Smirnov test; $n=10$ neurons per group) (Fig. $2 F$ ). The empirical distribution function for mEPSP amplitude in spared
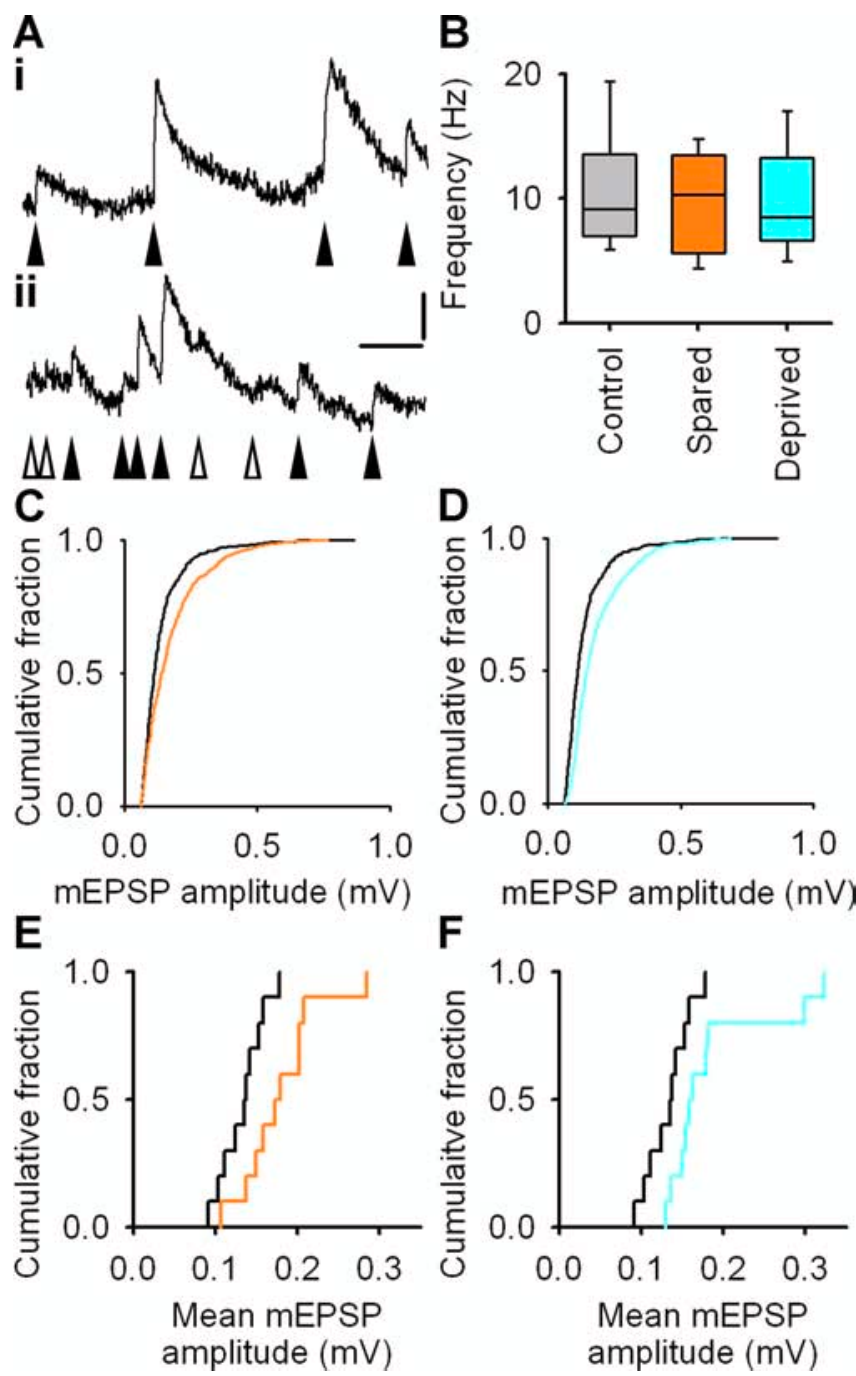

$\mathbf{F}$

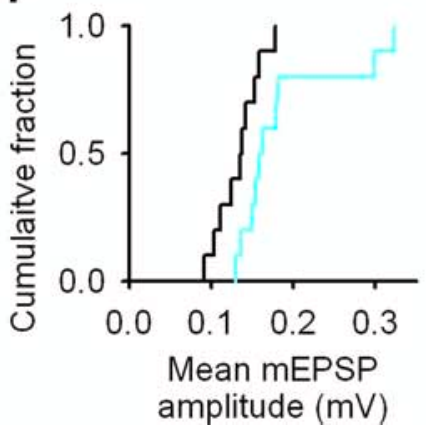

Figure 2. Miniature EPSPs are larger in spared and deprived cortex. $\boldsymbol{A}$, Example mEPSPs denoted by filled arrowheads. Open arrowheads point to depolarizations that do not fulfill our criteria for mEPSPs. Calibration: $0.2 \mathrm{mV}, 30 \mathrm{~ms}$. $\boldsymbol{B}$, Frequency of mEPSPs in control, spared, and deprived cortex. Box-and-whisker plots show median frequency and interquartile range with whiskers denoting the data range. $\boldsymbol{C}$, Empirical distribution plots of amplitudes of mEPSPs (50 $m$ mESPs per neuron) in spared (orange) and control (black) cortex. D, Empirical distribution plots of amplitudes of mEPSPs (50 mEPSPs per neuron) in deprived (cyan) and control (black) cortex. $\boldsymbol{E}$, Empirical distribution plots of mean mEPSP amplitudes in spared (orange) and control (black) cortex. F, Empirical distribution plots of mean mEPSP amplitudes in deprived (cyan) and control (black) cortex. 
cortex showed a similar rightward shift when compared with control cortex, but this did not reach statistical significance (Fig. $2 E)(p=0.11$, Kolmogorov-Smirnov test; $n=10$ neurons per group). The resting membrane potential and input resistance of neurons used for mEPSP analysis were similar in control, spared, and deprived cortex (data not shown), suggesting that our results were not attributable to differences in EPSP driving force or passive membrane properties of L2/3 pyramidal neurons.

Our recordings did not show the reduction in mEPSP frequency in deprived cortex that we had expected from the loss of local excitatory connectivity. We investigated this further by comparing mEPSPs with evoked unitary EPSPs (Fig. $1 \mathrm{Bi}$ ). The range of amplitudes spanned by the 0 to 95 th centiles of the control mEPSP distribution was $0.06-0.30 \mathrm{mV}$ (Fig. 2C,D). The mean percentage of evoked unitary EPSPs with amplitudes within this range was $41 \%$ ( $n=562$ unitary EPSPs from 16 control connections). Hence, many evoked unitary EPSPs were similar in amplitude to mEPSPs. However, more detailed analysis revealed that the vast majority of evoked unitary EPSPs in L2/3 pyramidal neurons were larger than $0.10 \mathrm{mV}$ ( 557 of 562 unitary EPSPs from 16 control connections). These findings indicated, first, that the smallest evoked unitary EPSPs were probably quantal responses and, second, that mEPSPs attributable to L2/3 inputs from neighboring pyramidal neurons were almost always larger than $0.10 \mathrm{mV}$. Many mEPSPs were smaller than this (Fig. $2 C, D)$. Hence, it is possible that we did not detect a decrease in mEPSP frequency in deprived cortex because it was obscured by variability introduced by small-amplitude $(<0.10 \mathrm{mV})$ mEPSPs. Therefore, we rethresholded our data to include only those mEPSPs larger than $0.10 \mathrm{mV}$ in amplitude. We refer to these datasets as L2/3-biased mEPSPs. The mean frequency of L2/3biased mEPSPs in either spared cortex $(7 \pm 1 \mathrm{~Hz} ; p=0.62$, Mann-Whitney rank sum test; $n=10$ neurons) or deprived cortex $(7 \pm 1 \mathrm{~Hz} ; p=0.66$, Mann-Whitney rank sum test; $n=10$ neurons) was not different from control cortex $(7 \pm 1 \mathrm{~Hz} ; n=10$ neurons). The mean amplitude of L2/3-biased mEPSPs in spared cortex $(0.22 \pm 0.01 \mathrm{mV} ; p=0.009, t$ test; $n=10$ neurons $)$ was greater than control cortex $(0.17 \pm 0.01 \mathrm{mV} ; n=10$ neurons $)$. The mean amplitude of L2/3-biased mEPSPs in deprived cortex $(0.20 \pm 0.02 \mathrm{mV} ; p=0.23$, Mann-Whitney rank sum test; $n=10$ neurons) was not statistically different from control values. This analysis supports the finding that mEPSP frequency was not reduced in deprived cortex despite loss of synaptic inputs from neighboring L2/3 pyramidal neurons.

The connectivity and mEPSP data together suggested that different processes were occurring in spared and deprived cortex. In spared cortex, the lack of change in local connectivity, unchanged mEPSP frequency, and increased mean mEPSP amplitude are consistent with strengthening of synapses without changes in synapse number. In deprived cortex, the preservation of mEPSP frequency in the presence of reduced local connectivity combined with the increased mean mEPSP amplitude in deprived cortex close to spared cortex pointed to a change in the source of excitatory inputs.

We explored the temporal and spatial development of the changes in mEPSPs in more detail with emphasis on deprived cortex. L2/3 pyramidal neurons in the $\mathrm{C}, \mathrm{D}$, and $\mathrm{E}$ barrel columns of the same brain slice were recorded after trimming the $\mathrm{D}$ and $\mathrm{E}$ whisker rows (Fig. 3A). In this experiment, whisker trimming started at P19 as before and continued for 13-20 d, which is shorter than the deprivation period used for the previous mEPSP study. Sham-trimmed rats served as controls. The frequency of mEPSPs in L2/3 of the spared C barrel column, the deprived D
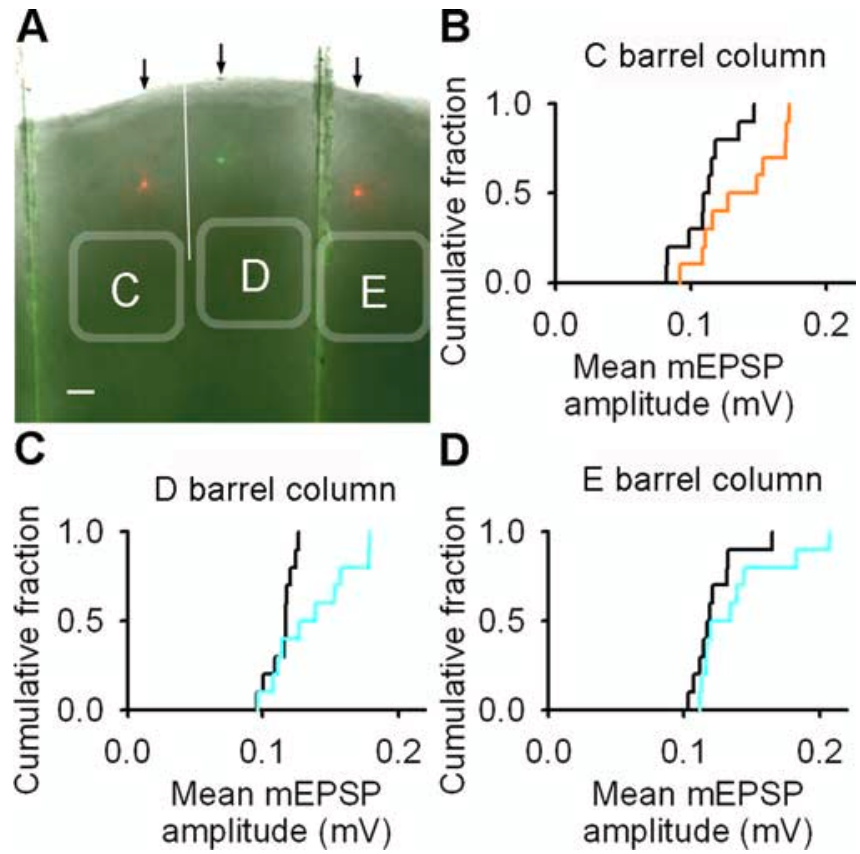

Figure 3. Changes in mEPSP amplitude in deprived cortex are greater close to the junction with spared cortex. $\boldsymbol{A}$, Image of a brain slice $(5 \times)$ with superimposed images of filled neurons in $\mathrm{L} 2 / 3$ of C (red soma), $D$ (green soma), and $E$ (red soma) barrel columns. White line denotes the border between C and D barrel columns. Scale bar, $100 \mu \mathrm{m}$. B, Empirical distribution plots of mean mEPSP amplitudes in the ( barrel column of control (black) and spared (orange) cortex. $\boldsymbol{C}$, Empirical distribution plots of mean mEPSP amplitudes in the D barrel column of control (black) and deprived (cyan) cortex. $\boldsymbol{D}$, Empirical distribution plots of mean mEPSP amplitudes in the $E$ barrel column of control (black) and deprived (cyan) cortex.

barrel column, and the deprived E barrel column were not different from the frequency of mEPSPs recorded from the corresponding barrel columns in control cortex (C-spared, $13 \pm 1 \mathrm{~Hz}$; C-control, $13 \pm 1 \mathrm{~Hz} ; p=0.79 ; \mathrm{D}$-deprived, $13 \pm 1 \mathrm{~Hz}$; D-control, $13 \pm 1 \mathrm{~Hz} ; p=0.99$; E-deprived, $12 \pm 1 \mathrm{~Hz}$; Econtrol, $14 \pm 1 \mathrm{~Hz} ; p=0.24, t$ tests; $n=10$ neurons for all groups). The mean amplitude of mEPSPs was larger in L2/3 of the spared C barrel column and the deprived D barrel column but not in the deprived $\mathrm{E}$ barrel column (C-spared, $0.14 \pm 0.01 \mathrm{mV}$; C-control, $0.11 \pm 0.01 \mathrm{mV} ; p=0.036, t$ test; $\mathrm{D}$-deprived, $0.14 \pm$ $0.01 \mathrm{mV}$; D-control, $0.11 \pm 0.01 \mathrm{mV} ; p=0.039$, $t$ test; E-deprived, $0.14 \pm 0.01 \mathrm{mV}$; E-control, $0.12 \pm 0.01 \mathrm{mV} ; p=$ 0.27 , Mann-Whitney rank sum test; $n=10$ neurons for all groups) (Fig. $3 B-D$ ). These data suggested that the synaptic changes in spared and deprived cortex were present after whiskers were trimmed for 13-20 d and that the increase in mEPSP amplitude in deprived cortex was most prominent at the junction of deprived and spared cortex.

Comparison of the results from animals that had been trimmed from P19 for 13-20 d with animals trimmed from P19 for 21-34 d suggested that mEPSP frequency decreased with age $\left(p=0.001 ; F_{(1,64)}=11.8\right.$; two-way ANOVA) but was not affected by deprivation status, i.e., spared, deprived, or sham trimmed ( $p=0.85 ; F_{(2,64)}=0.17$; two-way ANOVA). In contrast, mean mEPSP amplitude increased with age $(p<0.001$; $F_{(1,64)}=24.6$; two-way ANOVA) and deprivation status $(p<$ $0.001 ; F_{(2,64)}=8.5$; two-way ANOVA) (see Materials and Methods).

We investigated the effect of the age, deprivation status, and duration of deprivation on mean mEPSP amplitude under the generalized linear model framework. We first used the following 

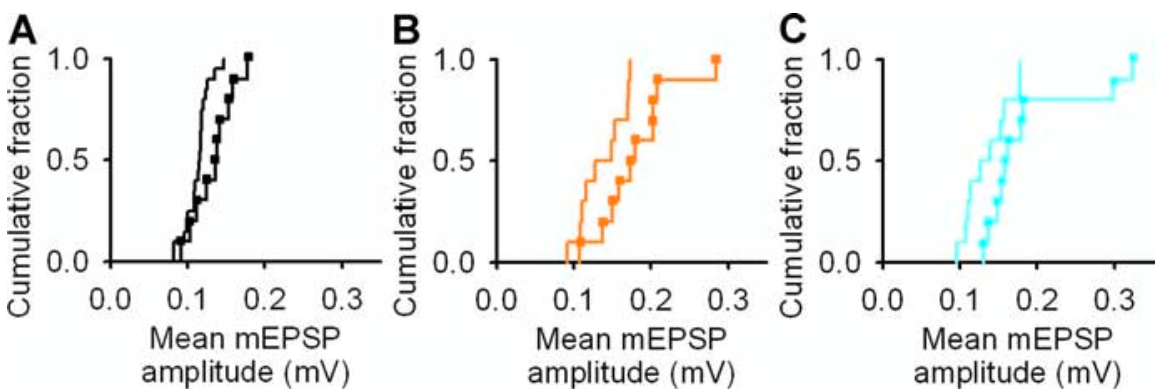

Figure 4. mEPSP amplitude increases with age and whisker deprivation. $\boldsymbol{A}$, Empirical distribution plots of mean $\mathrm{mEPSP}$ amplitudes of neurons in control cortex from rats aged either P32-P37 (black line) or P41-P53 (black line with squares). B, Empirical distribution plots of mean $m$ EPSP amplitudes of neurons in spared cortex from rats aged either P32-P39 (orange line) or P40 -P46 (orange line with squares). C, Empirical distribution plots of mean mEPSP amplitudes of neurons in deprived cortex from rats aged either P32-P39 (cyan line) or P40 -P46 (cyan line with squares).

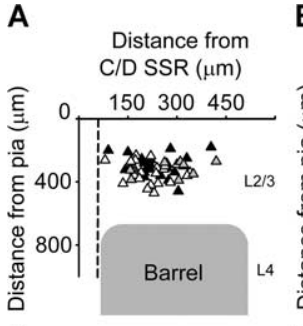

E

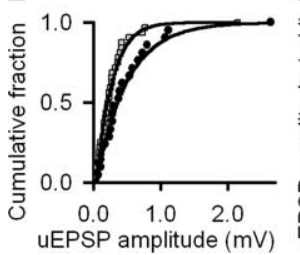

B
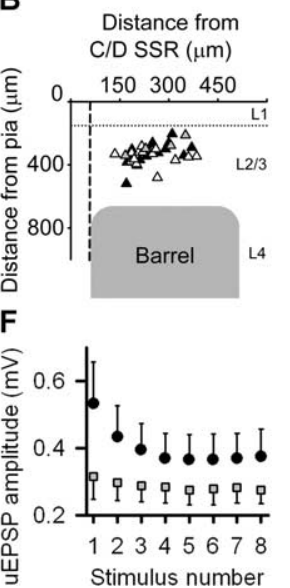

C

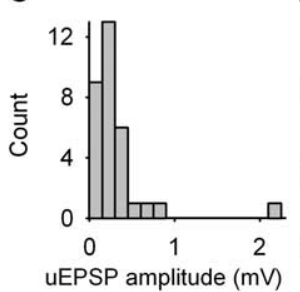

G

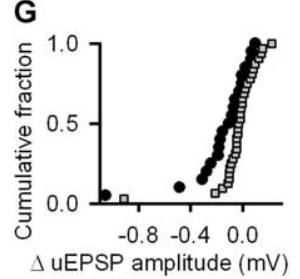

Figure 5. Local excitatory connections are stronger in spared cortex. A, Location of recorded pairs of synaptically connected neurons (presynaptic, black triangles; postsynaptic, white triangles) in control cortex. Gray triangles denote reciprocally connected neurons. Vertical dashed line represents the edge of the supraseptal region (SSR) separating $C$ and D barrel columns. Horizontal dotted line denotes the junction between layer 1 and layer 2. B, Location of recorded pairs of synaptically connected neurons in spared cortex. Symbols and lines as in A. C, Unitary EPSP (uEPSP) amplitudes in control cortex. D, Unitary EPSP amplitudes during $20 \mathrm{~Hz}$ stimulus trains may show (i) facilitation, (ii) mixed facilitation and depression, or (iii) depression. Traces are an average of 50 consecutive sweeps. Only one train of presynaptic action potentials is shown for simplicity. Calibration:50,0.1, 0.2, and $0.4 \mathrm{mV}$, $50 \mathrm{~ms}$. E, Empirical distribution plots of unitary EPSP amplitudes in spared (filled circles) and control (gray squares) cortex with the respective best fits by gamma distributions overlaid. $\boldsymbol{F}$, Mean unitary EPSP amplitude during $20 \mathrm{~Hz}$ trains in spared (filled circles) and control (gray squares) cortex. Error bars show SEM. G, Empirical distribution plots of changes in unitary EPSP amplitude at steady state during $20 \mathrm{~Hz}$ stimulus trains in spared (black circles) and control (gray squares) cortex. $\boldsymbol{H}$, Relationship between unitary EPSP amplitude (logarithmic scale) and age in spared (black circles) and control (gray squares) cortex. Linear regression fits (spared, solid line; control, dashed line) to the data are overlaid.

model: inverse $[E$ (mean mEPSP amplitude) $]=\alpha+\beta \times$ age + $\gamma \times \mathrm{DEP}+\delta \times \mathrm{SP}$, with an inverse link function and the Gaussian family (see Materials and Methods). Mean mEPSP amplitudes were described reasonably well by the model (null deviance, 0.129 ; residual deviance, $0.088 ; n=70$ neurons) and suggested that mEPSP amplitude increased with both age and deprivation status $(\alpha=13.1 \pm 1.6, p<0.001, t=8.3 ; \beta=-0.12 \pm 0.04, p=$ $0.001, t=-3.4 ; \gamma=-2.17 \pm 0.48, p<0.001, t=-4.1 ; \delta=$ $-1.92 \pm 0.55, p<0.001, t=-3.5$ ). We could not ascertain whether the effect of deprivation progressed during the deprivation protocol by incorporating interaction terms into this model because of collinearity. Therefore, we analyzed the mEPSP data using a second interaction model (inverse link function and the Gaussian family): inverse $[E$ (mean mEPSP amplitude) $]=\alpha+$ $\beta \times$ age $+\gamma \times$ DEP $\times$ age $+\delta \times \mathrm{SP} \times$ age. The interaction model did not result in a significant improvement in fit to the 山山山山ل'

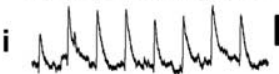
ii MWMAnl iii'Mumu! $\mathrm{H}$

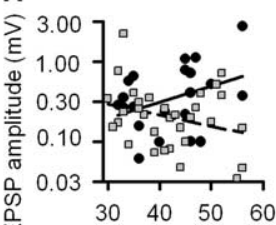

Age (postnatal days) data (residual deviance, 0.087) but was consistent with a progressive increase in mean mEPSP amplitude in spared and deprived cortex with longer deprivation $(\gamma=-0.053 \pm 0.013, p<0.001, t=$ $-4.24 ; \delta=-0.045 \pm 0.013, p<0.001$, $t=-3.47 ; \alpha=12.0 \pm 1.6, p<0.001, t=$ $8.0)$. The effect of deprivation was superimposed on a slow increase in $\mathrm{MEPSP}$ amplitude attributable to age alone $(\beta=$ $-0.09 \pm 0.04, p=0.011, t=-2.6)$.

Our analysis suggested that the increase in mean mEPSP amplitude in L2/3 pyramidal neurons has at least two components. First, there is a normal, slow increase in mEPSP amplitude between P32 and P53. This occurs in both shamtrimmed and whisker-trimmed animals. Superimposed on this normal increase is a second component attributable to deprivation, which further increases mEPSP amplitude at the border between spared and deprived cortex. The statistical models indicated that an effect of deprivation had developed by the time that we started recording and that this probably progressed over the recording period. In our animals, the deprivation component had a larger effect on mEPSP amplitude than the physiological increase with age [Kolmogorov-Smirnov tests, P32-P39 group compared with P40-P53 group: control, $p=$ 0.18 (Fig. $4 A$ ); spared, $p=0.001$ (Fig. $4 B$ ); deprived, $p=0.001$ (Fig. 4C)].

\section{Increased unitary EPSP amplitude in spared cortex}

Our data suggested strengthening of synapses in spared cortex but could not specify the origin of the strengthened inputs. L2/3 pyramidal neurons receive much of their excitatory input from neighboring pyramidal neurons (Binzegger et al., 2004). Therefore, we investigated whether sensory deprivation modified the EPSP amplitude at unitary connections between pairs of pyramidal neurons in the $\mathrm{C}$ or $\mathrm{D}$ barrel columns at the junction of deprived and spared cortex (see Figs. 5A, B, 6A). A change in unitary EPSP amplitude could be attributable to alterations in synapse strength or synapse number. Hence, we used the short-term synaptic dynamics at unitary connections as a second probe to assay whether there were functional changes at synapses. The mean baseline unitary EPSP amplitude in control cortex was $0.31 \pm 0.07 \mathrm{mV}$ ( $n=32$ connections) (Fig. 5C). During $20 \mathrm{~Hz}$ stimulation, unitary EPSPs facilitated, depressed, or showed a combination of facilitation and depression (Fig. 5D). In spared cortex, baseline unitary EPSP amplitudes $(0.53 \pm 0.12 \mathrm{mV} ; n=21)$ were larger than controls ( $p=0.027$, Mann-Whitney rank sum test). Unitary EPSP amplitudes in control and spared cortex were well described by gamma distributions $[\operatorname{control}(x ; 1.24,4.95,0.03), p=0.916 ; \operatorname{spared}(x ; 0.96,2.13$, $0.06), p=0.991$; Anderson-Darling test; see Materials and Methods] (Fig. 5E). The empirical distribution plot showed that uni- 
tary amplitudes in spared cortex were tilted toward higher values relative to controls ( $p<0.046$, two-sample Anderson-Darling test; see Materials and Methods). Moreover, unitary EPSPs in spared cortex depressed more during $20 \mathrm{~Hz}$ trains compared with controls (spared mean change, $-0.16 \pm 0.06 \mathrm{mV}, n=21$; control mean change, $-0.04 \pm 0.03 \mathrm{mV}, n=32 ; p=0.020$, MannWhitney rank sum test) (Fig. $5 F, G$ ). Despite the increased shortterm depression, excitatory drive was greater throughout $20 \mathrm{~Hz}$ trains (Fig. $5 F$ ). The empirical distribution plot of change in unitary EPSP amplitude at steady state showed a leftwards shift relative to controls ( $p=0.036$, Kolmogorov-Smirnov test) (Fig. $5 G)$, suggesting that there is greater depression at all unitary connections in spared cortex rather than a selective change in synaptic dynamics at a subpopulation of unitary connections. The ages of sham-trimmed rats and trimmed rats were not different (Table 1). There was no difference in the locations of presynaptic or postsynaptic neurons recorded in spared or control cortex (Fig. $5 A, B)$

We investigated whether the increase in unitary EPSP amplitude was affected by age and the duration of deprivation (Fig. $5 \mathrm{H}$ ) with the following model: $\ln$ (unitary EPSP amplitude) = $\alpha+\beta \times$ age $+\gamma \times$ age $\times$ SP, which used a natural log link function and Gaussian family (see Materials and Methods). Unitary EPSP amplitude in spared cortex increased with the duration of deprivation $(\delta=0.019 \pm 0.007 ; t=2.61 ; p=0.012)$ but was not affected by age in isolation $(\beta=0.033 \pm 0.021 ; t=1.61 ; p=$ $0.114)$. We concluded that synapses at local excitatory connections in spared cortex were stronger after deprivation and that this strengthening continued to develop over the deprivation period.

\section{Connection strength and synaptic dynamics do not alter in deprived cortex}

Unitary connections in deprived cortex gave very different results from spared cortex. The baseline unitary EPSP amplitude $(0.39 \pm$ $0.09 \mathrm{mV} ; p=0.220$, Mann-Whitney rank sum test; $n=8$ connections) (Fig. $6 \mathrm{~B}$ ), the change in EPSP amplitude at steady state (mean change, $-0.08 \pm 0.03 \mathrm{mV} ; p=0.106$, Mann-Whitney rank sum test) (Fig. 6C), and its distribution ( $p=0.18$, Kolmogorov-Smirnov test) (Fig. 6D) were not different from controls. The paucity of connections recorded from deprived cortex means that our results have to be interpreted with caution but indicate that there were no large-scale changes in the strength of the remaining local excitatory connections in deprived cortex.

\section{Synapse number is unchanged at local excitatory connections in spared cortex}

At least part of the increase in unitary EPSP amplitude and greater synaptic depression in spared cortex could have been attributable to an increase in synapse number per unitary connection with new synapses showing greater synaptic depression. Therefore, we used laser scanning confocal microscopy to reconstruct pairs of synaptically connected neurons filled with fluorescent dye during recording (Fig. $1 C$ ) and counted putative synapses between them (Fig. 7 $A, B$ ) (supplemental video, available at www.jneurosci.org as supplemental material) (see Materials and Methods).

We found no difference in the mean number of synapses per unitary connection in spared ( $3.0 \pm 0.9 ; n=8$ connections) and control ( $3.5 \pm 0.6 ; p=0.72$, $t$ test; $n=11$ connections $)$ cortex. The proportions of synapses on apical and basal dendrites formed by neighboring pyramidal neurons in spared cortex were not different from controls (apical:basal synapses, control, 22:16;
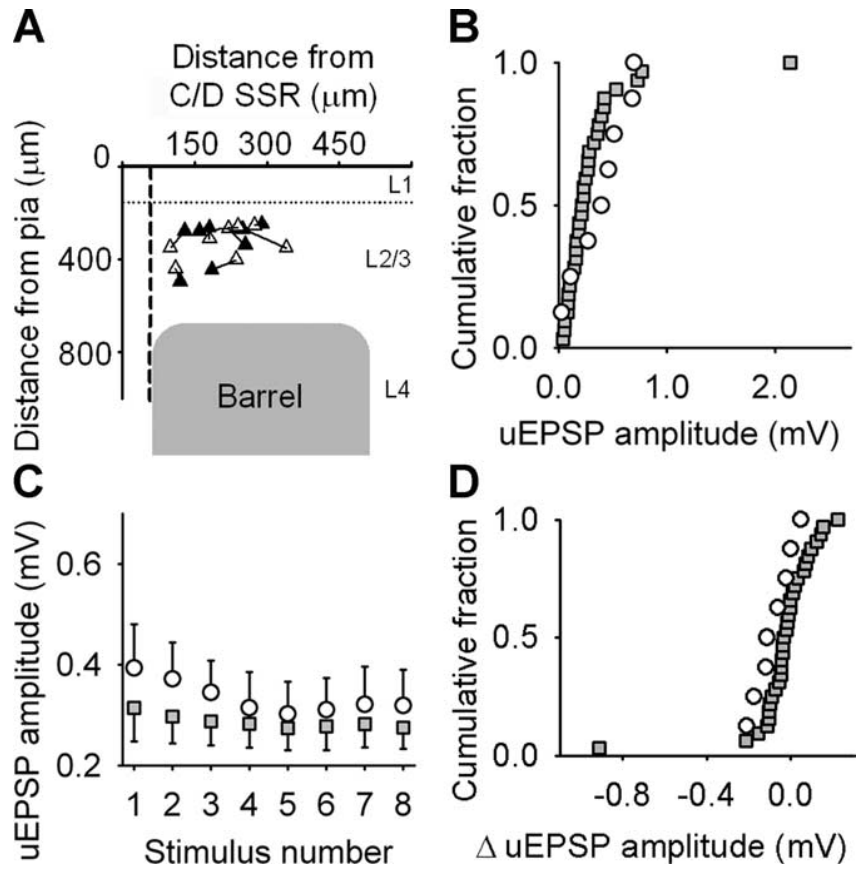

Figure 6. Unitary EPSP amplitude is unchanged in deprived cortex. $\boldsymbol{A}$, Location of recorded pairs of synaptically connected neurons in deprived cortex arranged as in Figure $5 A$. Presynaptic (black triangles) and postsynaptic (white triangles) neurons are connected by solid lines. $\boldsymbol{B}$ Empirical distribution plots of unitary EPSP (uEPSP) amplitudes in deprived (black circles) and control (gray squares) cortex. C, Mean unitary EPSP amplitude during $20 \mathrm{~Hz}$ trains in deprived (white circles) and control (gray squares) cortex. Error bars show SEM. D, Empirical distribution plots of changes in unitary EPSP amplitude at steady state during $20 \mathrm{~Hz}$ stimulus trains in deprived (white circles) and control (gray squares) cortex.

spared, $11: 13 ; \chi^{2}=0.44 ; \mathrm{df}=1 ; p=0.51$ ) (Fig. $7 C$ ). The locations of presynaptic and postsynaptic neurons used for synapse counts were representative of their respective groups (data not shown). We estimated the error associated with our optical criteria for identifying putative synapses by reconstructing either the unconnected direction from pairs of L2/3 pyramidal neurons that were unidirectionally connected $(n=8)$ or pairs of unconnected L $2 / 3$ pyramidal neurons $(n=2)$. The number of points of close proximity fulfilling our criteria for putative synapses was 0.17 per $\mathrm{L} 2 / 3$ axon $(n=12)$ unconnected to an adjacent $\mathrm{L} 2 / 3$ pyramidal neuron. This is $\sim 5 \%$ of the synapse number found for synaptically connected pairs of neurons. The cell bodies of unconnected neurons were separated by the same distance as the cell bodies of connected pairs (intersomatic distance: unconnected pair, $60 \pm 5 \mu \mathrm{m}$; connected pair, $63 \pm 5 \mu \mathrm{m} ; p=0.75$, MannWhitney rank sum test). Hence, the frequency of false-positive results returned by our optical criteria is within acceptable limits. We concluded that deprivation did not increase synapse number at connections between neighboring L2/3 pyramidal neurons in spared cortex.

We were able to reconstruct two pairs of synaptically connected $\mathrm{L} 2 / 3$ pyramidal neurons in deprived cortex, which formed a total of four putative synapses (Fig. 7C). The small sample size meant that we could not draw any reliable conclusions about structural reorganization in deprived cortex from these data.

\section{Discussion}

Our study shows that whisker deprivation induces site-specific modifications in local excitatory networks in primary somatosensory cortex. We found that there was a dramatic reduction in 

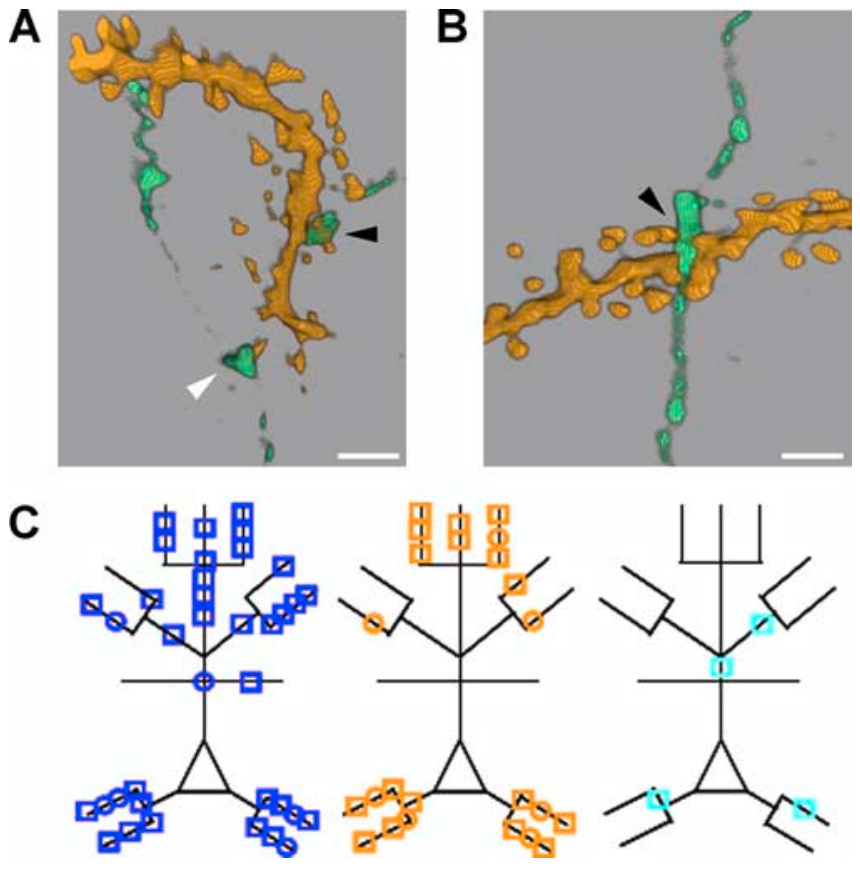

Figure 7. Deprivation does not alter synapse number at connections between pyramida neurons in spared cortex. $\boldsymbol{A}$, Volume-rendered (3D) reconstruction of a high-resolution confocal $z$-stack through a putative synapse (black arrowhead) onto a spine (supplemental video, available at www.jneurosci.org as supplemental material). The white arrowhead points at a varicosity that passes close $(\sim 0.6 \mu \mathrm{m})$ to a spine, but synaptic contact is not made. Scale bar, $1 \mu \mathrm{m}$. $\boldsymbol{B}$, Volume-rendered (3D) reconstruction of a putative synapse (black arrowhead) made onto a dendritic shaft. Scale bar, $1 \mu \mathrm{m}$. C, Schematic showing the locations of all putative synapses from reconstructed neurons in control cortex (blue, $n=38$ synapses from 11 connections), spared cortex (orange, $n=24$ synapses from 8 connections), and deprived cortex (cyan, $n=4$ synapses from 2 connections). Squares and circles denote axospinous and axodendritic synapses, respectively.

connectivity between $\mathrm{L} 2 / 3$ pyramidal neurons in deprived cortex, which was not accompanied by a compensatory increase in the strength of remaining local excitatory network connections. A markedly different situation occurred in adjacent spared cortex in which there was strengthening of connections between neighboring pyramidal neurons without a change in synapse number per connection or local excitatory connectivity.

Reduction of local excitatory connectivity in deprived cortex The precise cellular mechanisms underlying the marked reduction in connectivity that we report in deprived cortex remain to be elucidated. Our analysis indicated that the structural reorganization of local excitatory circuitry was well developed by P30. This made it difficult to be certain whether the reorganization was a global process affecting all connections or whether specific connections were targeted.

The lack of change in mEPSP frequency in deprived cortex suggests that one outcome of the reorganization process may be replacement of synaptic inputs previously formed by neighboring pyramidal neurons in deprived cortex by other excitatory inputs. Our recordings indicated that the increase in mean mEPSP amplitude was not uniform throughout deprived cortex but was greatest at the junction of deprived and spared cortex. Hence, one likely source for new excitatory synapses is horizontal intracortical L2/3 inputs from spared cortex, which have been reported to increase their axonal arborization into deprived cortex (Darian-Smith and Gilbert, 1994; Kossut and Juliano, 1999). These inputs strengthen after whisker deprivation (Finnerty et al., 1999). Therefore, it is possible that formation of new, stronger excitatory synapses by L2/3 inputs from spared cortex with pyramidal neurons in deprived cortex drives the loss of synapses between neighboring pyramidal neurons in deprived cortex. This hypothesis predicts that there should be increased synaptic inputs from L2/3 pyramidal neurons in spared cortex onto L2/3 pyramidal neurons in deprived cortex. We could not test this hypothesis directly because unitary connections between $\mathrm{L} 2 / 3$ pyramidal neurons in $\mathrm{C}$ and $\mathrm{D}$ barrel columns were difficult to find (no connections in 221 tests) in our brain slice preparation. This is not surprising given the distance $(>120 \mu \mathrm{m})$ between potential recording sites (Holmgren et al., 2003). Hence, additional work possibly combined with technical advances will be needed to test this prediction.

It has been proposed that synapse elimination in cortex resembles the process at immature neuromuscular junctions in which motor afferents compete to innervate a muscle cell (Lichtman and Colman, 2000). One of the motor inputs strengthens, with concurrent weakening of the other inputs before axon withdrawal of the weakened inputs (Colman et al., 1997). This hypothesis predicts that (1) unitary EPSP amplitudes of local excitatory connections in deprived cortex would decrease and (2) there would be a left shift in the empirical distribution function of unitary EPSP amplitudes compared with control unitary EPSP amplitudes. Our data do not support this hypothesis. However, the low connectivity in deprived cortex means that we found few connections. Hence, this hypothesis cannot be excluded and requires additional investigation with recordings before connections are lost.

The reduced connectivity in deprived cortex that we found is opposite to the increased connectivity in layer 4 of deprived visual cortex induced by monocular deprivation during the early critical period (Maffei et al., 2004). The difference in the results suggests that the effect of sensory deprivation on connectivity depends on several factors, including developmental status.

\section{Strengthening of local excitatory connections in spared cortex} Our data indicated that functional changes (progressive increase in unitary EPSP amplitude and greater synaptic depression) occurred at unitary connections between L2/3 pyramidal neurons in spared cortex without an alteration in the number of putative synaptic contacts. These findings suggest that whisker deprivation in developed animals primarily causes strengthening of excitatory unitary connections in spared cortex by modifying the properties of excitatory synapses at those connections rather than by increasing synapse number. The most parsimonious explanation for our results is that whisker trimming induced functional changes at existing synapses. The changes in unitary EPSP amplitude and synaptic dynamics that we found in spared cortex are similar to those that accompany long-term potentiation (LTP) induced by pairing presynaptic action potentials with postsynaptic depolarization in vitro (Markram and Tsodyks, 1996). This raises the possibility that sensory deprivation strengthens local excitatory connections in spared cortex in vivo by a process that resembles LTP in vitro. However, we cannot exclude the possibility that synapse turnover (Trachtenberg et al., 2002; Zuo et al., 2005a; De Paola et al., 2006; Stettler et al., 2006) results in new, stronger synapses being formed during the deprivation protocol.

Strengthening of local excitatory connections may play a role in cortical map expansion by either increasing firing within local excitatory networks in L2/3 of spared cortex or by altering the pattern of firing within those networks, e.g., by increasing synchronization. This would augment excitatory drive along hori- 
zontal intracortical pathways and, thereby, enhance expansion of spared representations into supragranular layers of deprived cortex (Das and Gilbert, 1995; Buonomano and Merzenich, 1998; Finnerty et al., 1999) or modify the representation of spared inputs within spared cortex (Diamond et al., 1994).

The effects of whisker deprivation on neuronal firing vary with the duration and pattern of deprivation (Fox, 2002). A commonly used method to study this involves mechanically displacing one whisker at low frequency. Evoked firing in L4 of a barrel column receiving its principal input from a spared whisker increases after whisker pairing (Armstrong-James et al., 1994) and chessboard deprivation (Wallace and Fox, 1999). Alterations in evoked principal whisker responses in L2/3 of the spared barrel column are not as prominent as those in L4 but include increased firing in a proportion of neurons (Barth et al., 2000; Wallace et al., 2001; Fox, 2002). In contrast, there is a robust increase in firing in L2/3 of deprived barrel columns (Glazewski and Fox, 1996; Wallace and Fox, 1999) and spared barrel columns (Diamond et al., 1994) that are adjacent to the barrel column receiving its principal input from the spared whisker. The latter finding suggests that there may be an interaction between spared whiskers. The magnitude of this effect in our preparation is not clear, but it raises the possibility that results from deprivation paradigms, which spare multiple whiskers, may not generalize to rodents with singlewhisker experience.

\section{Miniature EPSPs}

The effects of deprivation on mEPSP amplitude were superimposed on an age-dependent increase in mEPSP amplitude and decrease in mEPSP frequency. Spine density on neocortical neurons peaks between P15 and P30 before declining slowly (Wise et al., 1979; Markus and Petit, 1987; Zuo et al., 2005a). The decrease in mEPSP frequency that we found with age is consistent with the physiological spine loss that occurs in developed animals. The increase in mEPSP amplitude may reflect either loss of weaker synapses or strengthening of some synapses over our recording period.

The effects of deprivation on mEPSPs that we found in developed animals appear to differ from a study in the developing primary somatosensory cortex, which reported that mEPSC amplitudes in L2/3 pyramidal neurons after D-row deprivation were not different in the deprived $\mathrm{D}$ row compared with the spared $\mathrm{B}$ row (Bender et al., 2006). However, dissimilarities in the deprivation protocols make direct comparison difficult. In particular, experiments on visual cortex suggest that the timing of the deprivation protocol with respect to maturation of inputs to L4 has a marked effect on the outcome of deprivation-induced changes in mEPSCs recorded from L2/3 pyramidal neurons (Desai et al., 2002).

\section{Local excitatory circuitry and whisker representations}

We propose a simple model whereby changes in local excitatory networks could contribute to cortical map reorganization. This model incorporates the modifications in longer-distance horizontal connections reported previously (Finnerty et al., 1999) and complements findings from experiments on vertical intracortical connections (Finnerty et al., 1999; Allen et al., 2003; Bender et al., 2006; Clem and Barth, 2006). The alterations in responses at the junction of deprived and spared cortex induced by sensory deprivation are activity dependent (Wallace et al., 2001). In freely behaving rats, sensory deprivation is followed acutely by increased firing in spared cortex and decreased firing in deprived cortex (Kelly et al., 1999). We propose that the in- creased firing of pyramidal neurons in L2/3 of spared cortex has two effects. First, it results in strengthening of existing excitatory synapses in spared cortex, which manifests as potentiation of local excitatory connections. Second, increased firing or changes in the pattern of firing in $\mathrm{L} 2 / 3$ of spared cortex will augment the excitatory drive from spared to deprived cortex. We suggest that this promotes loss of excitatory synapses in deprived cortex. Hence, depression of sensory responses in deprived cortex and potentiation of sensory responses in spared cortex can be dissociated by manipulations that block synaptic potentiation (Glazewski et al., 1996) but do not affect the acute changes in firing. The apparently greater dependence of plasticity in deprived cortex on changes in wiring may explain why depression of deprived responses is abolished in adult animals, whereas potentiation of spared responses persists (Fox, 2002).

\section{References}

Allen CB, Celikel T, Feldman DE (2003) Long-term depression induced by sensory deprivation during cortical map plasticity in vivo. Nat Neurosci 6:291-299.

Andermann ML, Moore CI (2006) A somatotopic map of vibrissa motion direction within a barrel column. Nat Neurosci 9:543-551.

Armstrong-James M, Diamond ME, Ebner FF (1994) An innocuous bias in whisker use in adult rats modifies receptive fields of barrel cortex neurons. J Neurosci 14:6978-6991.

Barth AL, McKenna M, Glazewski S, Hill P, Impey S, Storm D, Fox K (2000) Upregulation of cAMP response element-mediated gene expression during experience-dependent plasticity in adult neocortex. J Neurosci 20:4206-4216.

Bender KJ, Allen CB, Bender VA, Feldman DE (2006) Synaptic basis for whisker deprivation-induced synaptic depression in rat somatosensory cortex. J Neurosci 26:4155-4165.

Binzegger T, Douglas RJ, Martin KA (2004) A quantitative map of the circuit of cat primary visual cortex. J Neurosci 24:8441-8453.

Brecht M, Roth A, Sakmann B (2003) Dynamic receptive fields of reconstructed pyramidal cells in layers 3 and 2 of rat somatosensory barrel cortex. J Physiol (Lond) 553:243-265.

Brumberg JC, Pinto DJ, Simons DJ (1999) Cortical columnar processing in the rat whisker-to-barrel system. J Neurophysiol 82:1808-1817.

Buonomano DV, Merzenich MM (1998) Cortical plasticity: from synapses to maps. Annu Rev Neurosci 21:149-186.

Chklovskii DB, Mel BW, Svoboda K (2004) Cortical rewiring and information storage. Nature 431:782-788.

Clem RL, Barth A (2006) Pathway-specific trafficking of native AMPARs by in vivo experience. Neuron 49:663-670.

Clements JD, Bekkers JM (1997) Detection of spontaneous synaptic events with an optimally scaled template. Biophys J 73:220-229.

Colman H, Nabekura J, Lichtman JW (1997) Alteration in synaptic strength preceding axon withdrawal. Science 275:356-361.

Darian-Smith C, Gilbert CD (1994) Axonal sprouting accompanies functional reorganization in adult cat striate cortex. Nature 368:737-740.

Das A, Gilbert CD (1995) Long-range horizontal connections and their role in cortical reorganization revealed by optical recording of cat primary visual cortex. Nature 375:780-784.

De Paola V, Holtmaat A, Knott G, Song S, Wilbrecht L, Caroni P, Svoboda K (2006) Cell type-specific structural plasticity of axonal branches and boutons in the adult neocortex. Neuron 49:861-875.

Desai NS, Cudmore RH, Nelson SB, Turrigiano GG (2002) Critical periods for experience-dependent synaptic scaling in visual cortex. Nat Neurosci 5:783-789.

Diamond ME, Huang W, Ebner FF (1994) Laminar comparison of somatosensory cortical plasticity. Science 265:1885-1888.

Fatt P, Katz B (1952) Spontaneous subthreshold activity at motor nerve endings. J Physiol (Lond) 117:109-128.

Feldman DE, Brecht M (2005) Map plasticity in somatosensory cortex. Science 310:810-815.

Feldmeyer D, Egger V, Lübke J, Sakmann B (1999) Reliable synaptic connections between pairs of excitatory layer 4 neurones within a single "barrel" of developing rat somatosensory cortex. J Physiol (Lond) 521:169-190. 
Finnerty GT, Roberts LSE, Connors BW (1999) Sensory experience modifies short-term dynamics of neocortical synapses. Nature 400:367-371.

Fox K (1992) A critical period for experience-dependent synaptic plasticity in rat barrel cortex. J Neurosci 12:1826-1838.

Fox K (2002) Anatomical pathways and molecular mechanisms for plasticity in the barrel cortex. Neuroscience 111:799-814.

Fox K, Wong RO (2005) A comparison of experience-dependent plasticity in the visual and somatosensory systems. Neuron 48:465-477.

Gilbert CD, Wiesel TN (1992) Receptive field dynamics in adult primary visual cortex. Nature 356:150-152.

Glazewski S, Fox K (1996) Time course of experience-dependent synaptic potentiation and depression in barrel cortex of adolescent rats. J Neurophysiol 75:1714-1729.

Glazewski S, Chen C-M, Silva A, Fox K (1996) Requirement for $\alpha$-CaMKII in experience-dependent plasticity of the barrel cortex. Science 272:421-423.

Hardingham NR, Larkman AU (1998) The reliability of excitatory synaptic transmission in slices of rat visual cortex in vitro is temperature dependent. J Physiol (Lond) 507:249-256.

Hensch TK (2004) Critical period regulation. Annu Rev Neurosci 27:549-579.

Holmgren C, Harkany T, Svennenfors B, Zilberter Y (2003) Pyramidal cell communication within local networks in layer $2 / 3$ of rat neocortex. J Physiol (Lond) 551:139-153.

Kelly MK, Carvell GE, Kodger JM, Simons DJ (1999) Sensory loss by selected whisker removal produces immediate disinhibition in the somatosensory cortex of behaving rats. J Neurosci 19:9117-9125.

Kim U, Ebner FF (1999) Barrels and septa: separate circuits in rat barrels field cortex. J Comp Neurol 408:489-505.

Kossut M, Juliano SL (1999) Anatomical correlates of representational map reorganization induced by partial vibrissectomy in the barrel cortex of adult mice. Neuroscience 92:807-817.

Lichtman JW, Colman H (2000) Synapse elimination and indelible memory. Neuron 25:269-278.

Maffei A, Nelson SB, Turrigiano GG (2004) Selective reconfiguration of layer 4 visual cortical circuitry by visual deprivation. Nat Neurosci 7:1353-1359.

Markram H, Tsodyks M (1996) Redistribution of synaptic efficacy between neocortical pyramidal cells. Nature 382:807-810.

Markus EJ, Petit TL (1987) Neocortical synaptogenesis, aging, and behavior: life-span development in the motor sensory system of the rat. Exp Neurol 96:262-278.

McCullagh P, Nelder JA (1989) Generalized linear models. London: Chapman and Hall.
Peters A, Kaiserman-Abramof IR (1969) The small pyramidal neuron of the rat cerebral cortex. Z Zellforschung 100:487-506.

Petersen CC, Grinvald A, Sakmann B (2003) Spatiotemporal dynamics of sensory responses in layer $2 / 3$ of rat barrel cortex measured in vivo by voltage-sensitive dye imaging combined with whole-cell voltage recordings and neuron reconstructions. J Neurosci 23:1298-1309.

Rioult-Pedotti MS, Friedman D, Hess G, Donoghue JP (1998) Strengthening of horizontal cortical connections following skill learning. Nat Neurosci 1:230-234.

Shepherd GM, Pologruto TA, Svoboda K (2003) Circuit analysis of experience-dependent plasticity in the developing rat barrel cortex. Neuron 38:277-289.

Simkus CR, Stricker C (2002) Properties of mEPSCs recorded in layer II neurones of rat barrel cortex. J Physiol (Lond) 545:509-520.

Song S, Sjostrom PJ, Reigl M, Nelson S, Chklovskii DB (2005) Highly nonrandom features of synaptic connectivity in local cortical circuits. PLoS Biol 3:e68.

Stephens MA (1986) Tests based on EDF statistics. In: Goodness-of-fit techniques (D’Agostino RB, Stephens MA, eds), pp 97-193. New York: Dekker.

Stern EA, Maravall M, Svoboda K (2001) Rapid development and plasticity of layer 2/3 maps in rat barrel cortex in vivo. Neuron 31:305-315.

Stettler DD, Yamahachi H, Li W, Denk W, Gilbert CD (2006) Axons and synaptic boutons are highly dynamic in adult visual cortex. Neuron 49:877-887.

Trachtenberg JT, Chen BE, Knott GW, Feng G, Sanes JR, Welker E, Svoboda $\mathrm{K}$ (2002) Long-term in vivo imaging of experience-dependent synaptic plasticity in adult cortex. Nature 420:788-794.

Wallace H, Fox K (1999) The effect of vibrissa deprivation pattern on the form of plasticity induced in rat barrel cortex. Somatosens Mot Res 16:122-138.

Wallace H, Glazewski S, Liming K, Fox K (2001) The role of cortical activity in experience-dependent potentiation and depression of sensory responses in rat barrel cortex. J Neurosci 21:3881-3894.

Wise SP, Fleshman Jr JW, Jones EG (1979) Maturation of pyramidal cell form in relation to developing afferent and efferent connections of rat somatic sensory cortex. Neuroscience 4:1275-1297.

Yoshimura Y, Dantzker JLM, Callaway EM (2005) Excitatory cortical neurons form fine-scale functional networks. Nature 433:868-873.

Zuo Y, Lin A, Chang P, Gan WB (2005a) Development of long-term dendritic spine stability in diverse regions of cerebral cortex. Neuron 46:181-189.

Zuo Y, Yang G, Kwon E, Gan WB (2005b) Long-term sensory deprivation prevents dendritic spine loss in primary somatosensory cortex. Nature 436:261-265. 